\title{
Facial Palsy and Nystagmus after Transvenous Embolization of a Carotid Cavernous Fistula
}

\author{
Seong-il Oh, ${ }^{a}$ Young Seo Kim, ${ }^{a}$ Young-Jun Lee, ${ }^{b}$ Hyeong-Joong Yi,,${ }^{c}$ Hyun Young Kim ${ }^{a}$ \\ Departments of ${ }^{\mathrm{a}}$ Neurology, ${ }^{\mathrm{b}}$ Radiology, and ${ }^{\mathrm{c} N e u r o s u r g e r y, ~ C o l l e g e ~ o f ~ M e d i c i n e, ~ H a n y a n g ~ U n i v e r s i t y, ~ S e o u l, ~ K o r e a ~}$
}

\section{Dear sir}

Intracranial dural carotid cavernous fistulas account for 10 to $15 \%$ of all intracranial arteriovenous malformations. ${ }^{1}$ Although these fistulas tend to regress spontaneously, permanent neurological deficits are observed in as many as $20-30 \%$ of untreated patients. ${ }^{2,3}$ In general, transvenous or transarterial embolization of the fistula is a safe and successful treatment option.

Depending on the amount and direction of venous drainage from the cavernous sinus, symptoms may include distended orbit, periorbital veins, and oculomotor, trochlear, and abducens nerve palsy. ${ }^{2,3}$ In addition, facial palsy and nystagmus are rarely seen as clinical manifestations of dural carotid cavernous fistulas, ${ }^{4}$ but can arise as complex consequences of embolization.

A 41-year-old female visited our clinic with headache and vertical diplopia. The headache was pulsatile, located in the right orbito-temporal region, and had persisted for a month. Other than bilateral myopia, the patient's medical history revealed no trauma, hypertension, diabetes, or other medical problems such as connective tissue disease. On admission, her vital signs were stable. Chest $\mathrm{X}$-ray and laboratory findings were normal. Neurological examination revealed vertical diplopia aggravated by upward gaze. When her head was tilted to the right side, the right eye showed hypotropia and vertical diplopia was aggravated. Thus, we confirmed the right superior rectus palsy; no other abnormalities were observed. Visual acuities and fields, pupil size and reactivity, and ophthalmoscopic findings were all normal. After a full examination for extraocular movement, right partial third cranial nerve palsy was suspected. The brain MRI revealed a carotid cavernous fistula (Figure 1A, B), and digital subtraction angiography confirmed a dural carotid cavernous fistula in the right cavernous sinus. The middle meningeal artery and internal maxillary arteries were the main feeders, and some branches of the meningohypophyseal trunk drained to the cavernous sinus. The main flow from the fistula and the sinus drained to the internal jugular vein through the inferior petrosal sinus. Hence the dural carotid cavernous fistula was classified as type $\mathrm{D}$ with branches of the internal carotid artery and the external carotid artery (Figure 1C, D). ${ }^{5}$

We first performed a transvenous intervention to the right cavernous sinus using coil embolization on the patient's third day in hospital. The transvenous embolization was performed under local anesthesia via the ipsilateral jugular vein; the vein was punctured and a sheath introduced. A catheter was easily navigated through the inferior petrosal sinus to the cavernous sinus. However, the moment the coil was placed in the anterior chamber of the cavernous sinus the patient complained of severe headache and the procedure was stopped. Immediately after the first partial embolization, right infranuclear facial palsy and left side mixed clockwise torsional nystagmus at a neutral position developed. The brain MRI was normal. Despite the partial embolization, no significant change in the drainage of the dural fistula was observed (Figure 1E, F), and no new cortical drainage developed. There were no arterial spasms or dissection.

In the second transarterial intervention on the sixth hospital day, arterial glue ( $20 \%$ n-butyl cyanoacrylate, $0.8 \mathrm{~mL})$ was injected into the middle meningeal artery. Injection into the internal maxillary artery was also attempted, but it was aborted because of arterial spasm. Eventually fistular flow as revealed by external carotid angiography was reduced (Figure 1G, H), and 

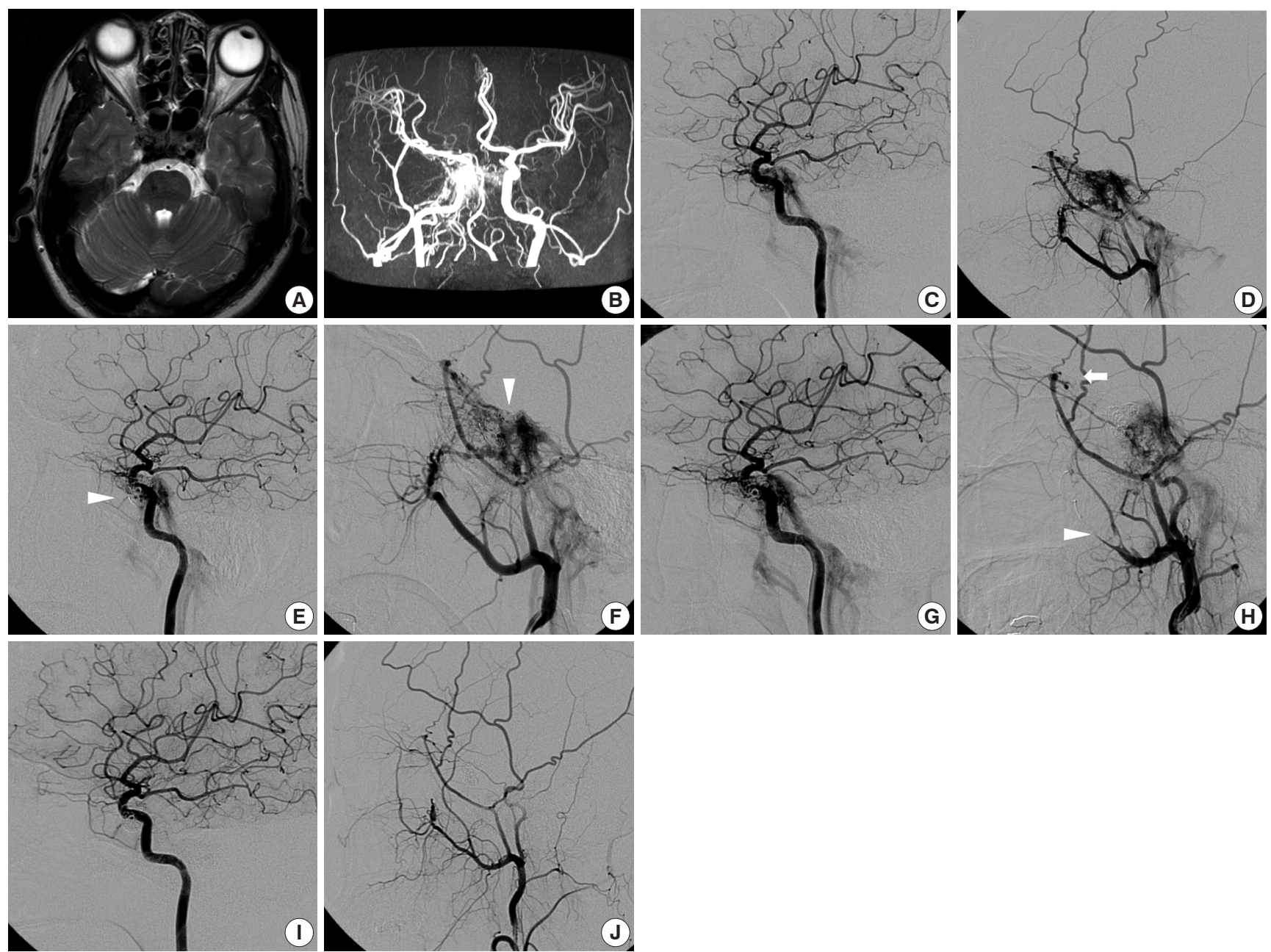

Figure 1. T2-weighted MRI (A) shows increased signal voids around right cavernous sinus and MR angiography (B) shows dural fistula flow from internal and external carotid arteries. Pre-embolization angiograms $(C, D)$ shows fistula flow via the internal carotid artery and the external carotid artery. The main flow drains to the internal jugular vein through the inferior petrosal sinus. Angiogram after the first transvenous embolization (E, F) shows insertion of the coil (arrowhead) into the cavernous sinus. After the second transarterial embolization $(\mathrm{G}, \mathrm{H})$, fistula flow via the middle meningeal artery (arrow) has decreased, and flow through the internal maxillary artery (arrowhead) has ceased due to vascular spasm. After 5 months, the dural carotid cavernous fistula has regressed (I, J) and there is no remnant aberrant flow.

the severity of the patient's headache and nystagmus decreased.

The patient's diplopia, nystagmus, and facial palsy gradually declined and resolved completely over two months. Subsequent angiography five months after embolization did not reveal any remnant of thedural carotid cavernous fistula (Figure 1I, J).

Symptoms of dural carotid cavernous fistula include proptosis, elevated intraocular pressure, pulsatile bruits, and less commonly, ophthalmoplegia, facial palsy, and facial pain. In the present case, facial palsy and nystagmus developed de novo after transvenousembolization. To the best of our knowledge, this is the first report of facial palsy and nystagmus occurring after transvenous embolization of a carotid cavernous fistula.

The classification of dural carotid cavernous fistulas by Barrow et al. involves four angiographic types, one direct (type $\mathrm{A}$ ) and three dural (types B, C, and D). ${ }^{5}$ Type D fistulas are shunts between the meningeal branches of both the internal carotid artery and external carotid arteryand the cavernous sinus. In the present case, the carotid cavernous fistula had multiple arteriovenous connections and was classified as type $\mathrm{D}$, prompting consideration of coiling via the transvenous approach.

The first intervention was not completed due to severe headache and the patient presented with facial nerve palsy and nystagmus shortly after transvenous embolization. We cannot fully account for the complications, but it is known that pressure palsy can occur because of slight venous engorgement around the internal auditory meatus connected to the inferior petrosal sinus, ${ }^{4}$ although no new apparent venous reflux was seen in our case. Moreover, because of the close proximity between the facial nerve and vestibulocochlear nerve, elevated venous pressure could affect the vestibulocochlear nerve and may be a cause 
of concomitant nystagmus. We carefully suggest such a possibility as a responsible cause of facial palsy and nystagmus after transvenous embolization in our case.

\section{References}

1. Quinones D, Duckwiler G, Gobin PY, Goldberg RA, Vinuela F. Embolization of dural cavernous fistulas via superior ophthalmic vein approach. AJNR Am J Neuroradiol 1997;18:921928.

2. Goldberg RA, Goldey SH, Duckwiler G, Vinuela F. Management of cavernous sinus-dural fistulas. Indications and techniques for primary embolization via the superior ophthalmic vein. Arch Ophthalmol 1996;114:707-714.

3. Kurata A, Takano M, Tokiwa K, Miyasaka Y, Yada K, Kan S. Spontaneous carotid cavernous fistula presenting only with cranial nerve palsies. AJNR Am J Neuroradiol 1993;14:10971101.
4. Kapur A, Sanghavi NG, Parikh NK, Amin SK. Spontaneous carotid-cavernous fistula with ophthalmoplegia and facial palsy. Postgrad Med J 1982;58:773-775.

5. Barrow DL, Spector RH, Braun IF, Landman JA, Tindall SC, Tindall GT. Classification and treatment of spontaneous carotid-cavernous sinus fistulas. J Neurosurg 1985;62:248-256.

\section{Correspondence: Hyun Young Kim} Department of Neurology, Hanyang University College of Medicine, 222 Wangsimri-ro, Seongdong-gu, Seoul 133-791, Korea Tel: +82-2-2290-8373

Fax: +82-2-2299-2391

E-mail: hyoungkim1@hanyang.ac.kr

Received: September 19, 2012 / Revised: December 26, 2012 Accepted: December 27, 2012

The authors have no financial conflicts of interest. 